\title{
ChILD - Children's interstitial lung disease
}

ChILD stands for children's interstitial lung disease. It is used to describe over 200 rare lung conditions that affect children.

This factsheet explains what ChILD is, and how it is diagnosed and managed.

More detailed information on the different forms of ChILD is available on the ChILD Patient Priorities website: www.europeanlunginfo.org/child

\section{What is an interstitial lung disease?}

The term 'interstitial lung disease' covers a large group of lung conditions that affect the interstitium, the tissue and space around the alveoli (air sacs) in the lungs. This is where oxygen is taken into the body and carbon dioxide is taken out of the body.

These conditions can affect the lungs in different ways. Most of the time, the walls of the interstitium get thicker, which makes it harder for the lungs to expand (when breathing in) or contract (when breathing out). This can lead to lower levels of oxygen in the blood and may make a child short of breath. To make up for this they will often breathe faster, which uses up more energy and makes them very tired.

ChILD also includes some conditions whose effects are more widespread in the lungs and are not just focused on the interstitium. These are called diffuse lung diseases. The symptoms and effects on the body are usually the same, which is why they are known as ChILD conditions.

ChILD conditions are linked to a range of potential causes, including the genes a child inherits from their parents, having problems with the immune system, being exposed to air pollution, and going through chemotherapy or radiotherapy.

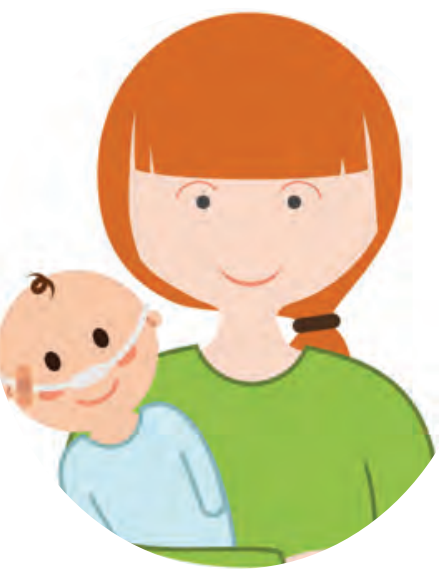

\section{What are the signs and symptoms?}

Here are some of the main signs and symptoms of ChILD:

- Being short of breath

- Breathing very quickly

- Difficult and heavy breathing 
- Getting tired more easily than children their age, e.g. needing to stop a lot when walking, difficulty climbing stairs

- Not gaining weight or losing weight (also called failure to thrive)

- Breathing noises, e.g. rattles, crackles or wheezing

- Coughing a lot

- Having blue or grey lips

- Clubbing of the fingers (where the fingertips become spoon-shaped) and hourglass nails (where the fingernails bulge into a rounded shape)

Not every child will have all of these symptoms - and having these symptoms does not mean your child has ChILD. If your child has these symptoms, contact your doctor and explain your concerns.

\section{Steps to diagnosis}

As ChILD conditions are very rare, they can be hard to diagnose. When ChILD is first suspected, your child's condition should ideally be discussed with a specialist with experience of diagnosing and managing these rare conditions.

There are several investigations that can help diagnose ChILD. These range from physical examinations, breathing tests and blood tests, to more detailed tests such as CT (computer tomography) scans, bronchoalveolar lavages (washes) and lung biopsies. Some ChILD conditions can also be diagnosed with a genetic blood test.

Piecing together bits of information and considering how a condition develops over time is often how ChILD is diagnosed. This takes time and can be frustrating. Often there is a 'working diagnosis' that needs more information to make a firm diagnosis. A diary of symptoms, investigations and treatments already completed can be a helpful resource to take with you to appointments. This can help to get a successful diagnosis.

\section{How will it develop over time?}

In most cases, ChILD is a long-term condition and children may have lower lung function in adulthood, but this is not the same for everyone. Children can learn to live with their condition and often improve each year as they become more able to exercise.

Being uncertain about what lies ahead can be very difficult. Parents of children with ChILD say it is helpful to think in small steps; to take comfort in each improvement and to not despair if there is a setback. 
"My advice to other parents is something that another parent told me. When I am having a really tough time, I have to pull myself back to it: to live in the moment and not think too far ahead. This is difficult, but means you can make the most of every day."

\section{Kerry, whose son Noah has been diagnosed with ChILD}

\section{Treatment}

There is no single treatment for all types of ChILD and there is currently no cure for any of the conditions. However, many children grow out of their illness.

Most treatments used to manage ChILD are well-known and proven to work for other conditions. These include:

\section{Oxygen therapy}

Some children will benefit from supplementary oxygen (oxygen therapy) to help them get enough oxygen into their body.

\section{Medication}

Some medications that may be prescribed include steroids, other anti-inflammatory drugs or antibiotics. Your healthcare professional will advise on which medication might be most helpful for your child's particular condition. Not every child will need medication.

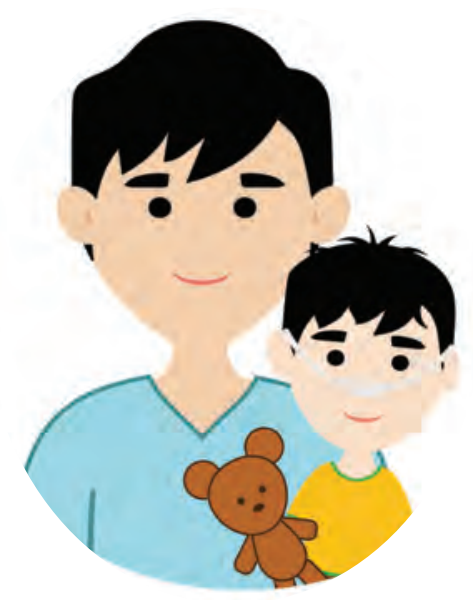

\section{Lung transplantation}

A lung transplant may be considered in very rare and severe cases when a child's health keeps getting worse despite medical care - and only after all other treatment options have been tried.

\section{Daily living}

Looking after your child may feel like a big responsibility in the early days. Taking up any support on offer can really help. There are a few important things to be aware of:

\section{Feeding and nutrition}

Children with ChILD often need more calories than other children because their increased breathing rate means they use up a lot of energy. When weight gain is good, your child is likely to be in relatively good health. Weight loss and poor growth are serious warning signs of an underlying problem, so you should talk to your doctor if this happens. 


\section{Everyday illness}

Children with ChILD do not usually get ill with infections more often than healthy children (unless they have a condition which means their immune system does not work properly, called a primary immunodeficiency; are on steroids; or take other drugs that affect the immune system), but they have a higher risk of becoming more severely ill with them. Your healthcare professionals will be able to give you advice after carefully weighing up

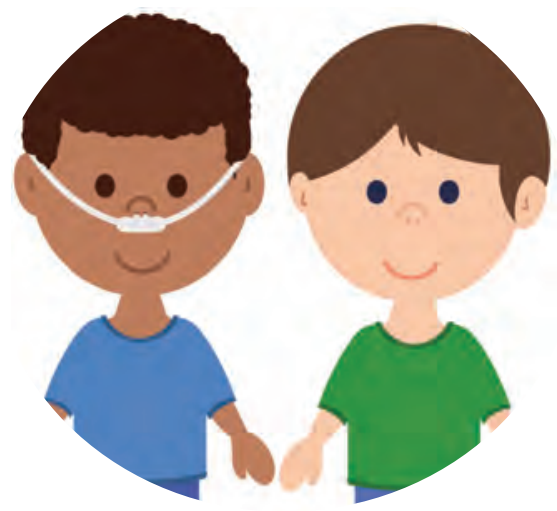
the potential risks and benefits to your child of taking part in activities. No measure can completely protect your child from infection and stopping them from taking part in activities with other children could significantly reduce their quality of life.

\section{Education and recreation}

Most children with ChILD can go to nursery or school - and it is important that they do. Carers and teachers should be given information about your child's condition and what they must do in an emergency.

Sport and exercise should also be encouraged, as it has a positive impact on lung health, as well as social benefits. It is important to make sure that children are encouraged to take part, do not have to be scored or graded, and can rest if they need to.

\section{Social and financial support}

You may be able to get financial and practical support from the government in your country to help you manage. Find out what you are entitled to and make a claim as soon as possible. There may also be are and respite services that you can access for your child or for yourself - sometimes having a short break can help with the daily challenges.

\section{Further reading}

- ChILD Patient Priorities | www.europeanlunginfo.org/child Information and support for parents, caregivers and family members of children with ChILD, developed with the help of carers and healthcare professionals across Europe. A more detailed version of this information - as well as other resources, parent stories, and information on patient organisations and support groups - is available on the website.

- European Lung Foundation | www.europeanlung.org For more information on lung conditions and how to look after your lungs.

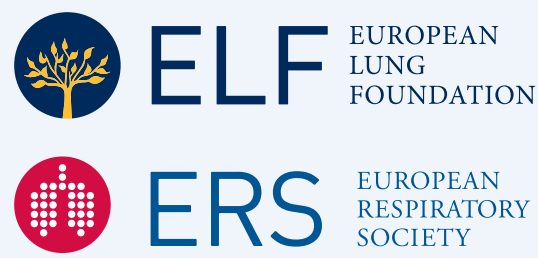

The European Lung Foundation (ELF) was founded by the European Respiratory Society (ERS) in 2000 with the aim of bringing together patients, the public and respiratory professionals to positively influence lung health.

This material was compiled as part of the ChILD patient priorities project with the help of Professor Steve Cunningham, Professor Matthias Griese Dr Nicolaus Schwerk and members of ELF's ChILD parent advisory group. Produced in November 2018. 